\title{
WHITEHEAD GROUPS OF EXCHANGE RINGS WITH PRIMITIVE FACTORS ARTINIAN
}

\author{
HUANYIN CHEN and FU-AN LI
}

(Received 8 October 2000)

\begin{abstract}
We show that if $R$ is an exchange ring with primitive factors artinian then $K_{1}(R) \cong U(R) / V(R)$, where $U(R)$ is the group of units of $R$ and $V(R)$ is the subgroup generated by $\left\{(1+a b)(1+b a)^{-1} \mid a, b \in R\right.$ with $\left.1+a b \in U(R)\right\}$. As a corollary, $K_{1}(R)$ is the abelianized group of units of $R$ if $1 / 2 \in R$.
\end{abstract}

2000 Mathematics Subject Classification. 16E50, $19 B 10$.

Very recently, Ara et al. [2] showed that the natural homomorphism $\mathrm{GL}_{1}(R) \rightarrow K_{1}(R)$ is surjective provided that $R$ is a separative exchange ring. A natural problem is the description of the kernel of the epimorphism $\mathrm{GL}_{1}(R) \rightarrow K_{1}(R)$. In [9, Theorems 1.2 and 1.6], Menal and Moncasi showed that if $R$ satisfies unit 1-stable range or is unitregular, then $K_{1}(R) \cong U(R) / V(R)$. Here $U(R)$ is the group of units of $R$ while $V(R)$ is a subgroup described later. In [7], Goodearl and Menal remarked that if for each $x, y \in R$ there exists a unit $u \in R$ such that $x-u$ and $y-u^{-1}$ are both units, then $K_{1}(R) \cong U(R)^{a b}$. In this paper, we investigate the above kernel for exchange rings with primitive factors artinian.

Recall that $R$ is called an exchange ring if for every right $R$-module $A$ and two decompositions $A=M \oplus N=\oplus_{i \in I} A_{i}$, where $M_{R} \cong R$ and the index set $I$ is finite, there exist submodules $A_{i}^{\prime} \subseteq A_{i}$ such that $A=M \oplus\left(\oplus_{i \in I} A_{i}^{\prime}\right)$. It is well known that regular rings, $\pi$-regular rings, semiperfect rings, left or right continuous rings, clean rings and unit $C^{*}$-algebras of real rank zero [1] are all exchange rings.

Many authors have studied exchange rings with primitive factors artinian. Fisher and Snider proved that every regular ring with primitive factors artinian is unitregular (see [6, Theorem 6.10]). Moreover, Menal [8, Thereom B] proved that every $\pi$-regular ring with primitive factors artinian has stable range one. Recently, Yu [13, Thereom 1] extended these results to exchange rings and showed that every exchange ring with primitive factors artinian has stable range one. On the other hand, Pardo [10] investigated the Grothendieck group $K_{0}$ of exchange rings. In this paper, we show that the Whitehead group $K_{1}(R) \cong U(R) / V(R)$ for an exchange ring $R$ with primitive factors artinian. We refer the reader to [11] for the general theory of Whitehead groups.

Throughout this paper, all rings are associative with identity. The set $U(R)$ denotes the set of all units of $R, V(R)$ denotes the subgroup generated by $\left\{p(a, b) p(b, a)^{-1}\right.$ | $p(a, b) \in U(R), a, b \in R\}$, and $J(R)$ denotes the Jacobson radical of $R$ (see below for the definition of $p(a, b)$ and other continuant polynomials). If $G$ is a group and $G^{\prime}$ its 
commutator subgroup, then $G^{a b}$ denotes $G / G^{\prime}$. Let $\mathrm{GL}_{n}(R)$ be the group of units of $M_{n}(R)$, the ring of all $n \times n$ matrices over $R$.

We start with the following new element-wise property of exchange rings with primitive factors artinian.

LEMMA 1. Let $R$ be an exchange ring with primitive factors artinian. Then, for any $x, y \in R$, there exists a unit-regular $w \in R$ such that $1+x y-x w \in U(R)$.

Proof. Assume that there are some $x, y \in R$ such that $1+x y-x w \notin U(R)$ for any unit-regular $w \in R$. Let $\Omega$ be the set of all two-sided ideals $A$ of $R$ such that $1+x y-x w$ is not a unit modulo $A$ for any unit-regular $w+A \in R / A$. Clearly, $\Omega \neq \varnothing$.

Given any ascending chain $A_{1} \subseteq A_{2} \subseteq \cdots \subseteq A_{n} \subseteq \cdots$ in $\Omega$, set $M=\cup_{1 \leq i \leq \infty} A_{i}$. Then $M$ is a two-sided ideal of $R$. Assume that $M$ is not in $\Omega$. Then there exists a unitregular $w+M \in R / M$ such that $(1+x y-x w)+M$ is a unit of $R / M$. Since $w+M$ is unit-regular in $R / M$, we have $e+M=(e+M)^{2}, u+M \in U(R / M)$ such that $w+M=$ $(e+M)(u+M)$. As $R / M$ is also exchange, we may assume that $e=e^{2} \in R$. Thus we can find positive integers $n_{i}(1 \leq i \leq 5)$ such that $(1+x y-x w) s-1 \in A_{n_{1}}, s(1+x y-$ $x w)-1 \in A_{n_{2}}, w-e u \in A_{n_{3}}, u t-1 \in A_{n_{4}}$, and $t u-1 \in A_{n_{5}}$ for some $s, t \in R$. Let $n=\max \left\{n_{1}, n_{2}, n_{3}, n_{4}, n_{5}\right\}$. Then $1+x y-x w+A_{n}$ is a unit of $R / A_{n}$ for a unit-regular $w+A_{n} \in R / A_{n}$. This contradicts the choice of $A_{n}$. So $M \in \Omega$. That is, $\Omega$ is inductive. By using Zorn's lemma, we have a two-sided ideal $Q$ of $R$ such that it is maximal in $\Omega$.

Let $S=R / Q$. If $J(R / Q) \neq 0$, then $J(R / Q)=K / Q$ for some $K \supset Q$. Clearly, $S / J(S) \cong$ $R / K$. By the maximality of $Q$, we can find a unit-regular $(v+Q)+J(S)$ such that $((1+x y-x v)+Q)+J(S)$ is a unit of $S / J(S)$. Since idempotents and units of $S / J(S)$ can be lifted modulo $J(S)$, we may assume that $v+Q$ is unit-regular in $S$. On the other hand, $(1+x y-x v)+Q=(m+Q)+(r+Q)$ for some $m+Q \in U(S), r+Q \in J(S)$. Thus, $(1+x y-x v)+Q$ is a unit of $S$. This gives a contradiction, whence $J(R / Q)=0$.

By the maximality of $Q$, one easily checks that $R / Q$ is an indecomposable ring. According to [14, Lemma 3.7], $R / Q$ is a simple artinian ring. Clearly, $(1+x y-x y)+$ $Q=1+Q$ is a unit of $R / Q$. Since $R / Q$ is unit-regular, $y+Q$ is a unit-regular element of $R / Q$. This contradicts the choice of $Q$. Therefore the proof is complete.

We note that the following conditions for a ring $R$ are equivalent.

(1) For any $x, y \in R$, there exists unit-regular $w \in R$ such that $1+x(y-w) \in U(R)$.

(2) Given $a R+b R=R$, then $a+b w \in U(R)$ for some unit-regular $w \in R$.

(3) Given $a x+b=1$, then $a w+b \in U(R)$ for some unit-regular $w \in R$ (cf. [5, Theorem 2.1]).

Clearly, the conditions above are stronger than the stable range one condition and can be viewed as a generalization of rings with many idempotents [4]. Call $R$ a ring with many unit-regular elements if the equivalent conditions above hold. In [5, Theorem 3.1], the authors showed that such rings are all $G E_{2}$. Furthermore, Chen [3] proved that if $R$ has many unit-regular elements then so does $M_{n}(R)$. Now we include the fact that every exchange ring with primitive factors artinian has many unit-regular elements to make our paper self-contained.

Recall that $p(a)=a, p(a, b)=1+a b$, and $p(a, b, c)=a+c+a b c$ for any $a, b, c \in R$. It is easy to verify that $p(a, b, c)=p(a, b) c+p(a), p(a, b, c) p(b, a)=p(a, b) p(c, b, a)$. 
LEMmA 2. Let $a, b, c \in R$ with $p(a, b, c) \in U(R)$. If $a$ is unit-regular, then $p(a, b, c)$ $\equiv p(c, b, a)(\bmod V(R))$.

Proof. Since $a$ is unit-regular, there is an idempotent $e$ and a unit $u$ such that $a=e u$. So we have $p(a, b, c)=e u+c+e u b c$, and then $p(a, b, c) u^{-1}=e+c u^{-1}+$ $e u b c u^{-1}$. Obviously, $p(e,-u b(1-e))=p(e,-u b(1-e))(p(-u b(1-e), e))^{-1} \in V(R)$. Thus we see that

$$
\begin{aligned}
p(a, b, c) u^{-1} & \equiv p(e,-u b(1-e)) p(a, b, c) u^{-1} \quad(\bmod V(R)) \\
& =(1-e u b(1-e))\left(e+c u^{-1}+e u b c u^{-1}\right) \\
& =e+c u^{-1}+e u b e c u^{-1} \quad(\bmod V(R)) .
\end{aligned}
$$

On the other hand, we can verify that

$$
\begin{aligned}
p(c, b, a) u^{-1} & =e+c u^{-1}+c b e \equiv\left(e+c u^{-1}+c u^{-1} u b e\right) p(1-e,-u b e) \quad(\bmod V(R)) \\
& =e+c u^{-1}+c u^{-1} e u b e=1+\left(c u^{-1}-(1-e)\right)(1+e u b e) \\
& \equiv 1+(1+e u b e)\left(c u^{-1}-(1-e)\right) \quad(\bmod V(R)) \\
& \equiv p(a, b, c) u^{-1} \quad(\bmod V(R)) .
\end{aligned}
$$

Consequently, $p(a, b, c) \equiv p(c, b, a)(\bmod V(R))$, as asserted.

In [4, Theorem 16], Chen showed that $K_{1}(R) \cong U(R) / V(R)$ provided that $R$ has idempotent 1-stable range. Now we extend this fact to exchange rings with primitive factors artinian.

THEOREM 3. Let $R$ be an exchange ring with primitive factors artinian. Then $K_{1}(R)$ $\cong U(R) / V(R)$.

Proof. Given $a, b, c \in R$ with $p(a, b, c) \in U(R)$, we have $p(c, b, a) \in U(R)$. From Lemma 1 , we can find some unit-regular $w \in R$ such that $1+b(c-w) \in U(R)$. Let $c-w=t$. Then $c=t+w$ and $1+b t \in U(R)$. We check that

$$
\begin{aligned}
p(a, b, c) & =a+c+a b c=(a+t+a b t)+(1+a b) w \\
& =(a+t+a b t)+(1+a b+t b+a b t b)(1+t b)^{-1} w \\
& =(a+t+a b t)+(1+t b)^{-1} w+(a+t+a b t) b(1+t b)^{-1} w \\
& =(1+t b)^{-1}\left((1+t b)(a+t+a b t)+w+(1+t b)(a+t+a b t) b(1+t b)^{-1} w\right) \\
& =(1+t b)^{-1} p\left((1+t b)(a+t+a b t), b(1+t b)^{-1}, w\right) .
\end{aligned}
$$

In view of Lemma 2, we know that

$$
\begin{aligned}
p(a, b, c) & \equiv(1+t b)^{-1} p\left(w, b(1+t b)^{-1},(1+t b)(a+t+a b t)\right) \quad(\bmod V(R)) \\
& =(1+t b)^{-1}\left(p\left(w, b(1+t b)^{-1}\right)(1+t b)(a+t+a b t)+p(w)\right) \\
& =(1+t b)^{-1}\left(p\left(w, b(1+t b)^{-1}\right) p(t, b) p(a, b, t)+p(w)\right) \\
& =(1+t b)^{-1}\left(p\left(w, b(1+t b)^{-1}\right) p(t, b, a) p(b, t)+p(w)\right) .
\end{aligned}
$$


On the other hand,

$$
\begin{aligned}
p(c, b, a) & =p\left(w,(1+b t)^{-1} b,(t+a+t b a)(1+b t)\right)(1+b t)^{-1} \\
& =\left(p\left(w,(1+b t)^{-1} b\right)(t+a+t b a)(1+b t)+p(w)\right)(1+b t)^{-1} \\
& =\left(p\left(w,(1+b t)^{-1} b\right) p(t, b, a) p(b, t)+p(w)\right)(1+b t)^{-1} .
\end{aligned}
$$

Since $b(1+t b)^{-1}=(1+b t)^{-1} b,(1+t b) p(a, b, c) \equiv p(c, b, a)(1+b t)(\bmod V(R))$. Hence $p(a, b, c)(p(c, b, a))^{-1} \in V(R)$ because $U(R) / V(R)$ is abelian. By virtue of [13, Theorem 1], $R$ has stable range one. It follows from [9, Theorem 1.2] that $K_{1}(R) \cong$ $U(R) / W(R) \cong U(R) / V(R)$, where the notation $W(R)$ denotes the subgroup of $U(R)$ generated by $\left\{p(a, b, c) p(c, b, a)^{-1} \mid p(a, b, c) \in U(R), a, b, c \in R\right\}$. So the proof is complete.

Recall that an exchange ring is said to be of bounded index if there exists some positive integer $n$ such that $x^{n}=0$ for all nilpotent $x \in R$. We can derive the following corollary.

COROLLARY 4. Let $R$ be an exchange ring of bounded index. Then $K_{1}(R) \cong \mathrm{GL}_{3}(R)^{a b}$ $\cong U(R) / V(R)$.

Proof. It is easy to obtain the first isomorphism by an argument of Menal. In view of [13, Theorem 3], $R$ is an exchange ring with primitive factors artinian. Therefore we complete the proof by Theorem 3 .

Write $L_{1}(R)$ for the subgroup generated by the elements in $U(R)^{\prime} \cup L$, where $L$ is the subgroup generated by all $1+e r(1-e)$ with $e=e^{2}, r \in R$. Clearly, $U(R)^{\prime} \subseteq L_{1}(R) \subseteq V(R)$.

LEMMA 5. Let $a, b \in R$ with $1+a b \in U(R)$. If $a$ is unit-regular, then $p(a, b) \equiv$ $p(b, a)\left(\bmod L_{1}(R)\right)$.

Proof. Since $a$ is unit-regular, there is an idempotent $e$ and a unit $u$ such that $a=u e$. So we have

$$
\begin{aligned}
p(a, b) & =1+u e b=u(1+e b u) u^{-1} \\
& =u(1+e b u) u^{-1}(1+e b u)^{-1}(1+e b u) \\
& \equiv(1+e b u) \quad\left(\bmod L_{1}(R)\right) \\
& \equiv(1-e b u(1-e))(1+e b u) \quad\left(\bmod L_{1}(R)\right) \\
& =1+e b u e=(1+b u e)(1-(1-e) b u e) \\
& \equiv(1+b u e) \quad\left(\bmod L_{1}(R)\right)=p(b, a), \quad \text { as required. }
\end{aligned}
$$

LEMMA 6. Let $R$ be an exchange ring with primitive factors artinian. If $R$ does not have $\mathbb{Z} / 2 \mathbb{Z}$ as a homomorphic image, then, for any $x, y \in R$, there exists a $w \in U(R)$ such that $1+x(y-w) \in U(R)$ and $y-w$ is unit-regular.

Proof. Assume that there exist some $x, y \in R$ such that $1+x(y-w) \notin U(R)$ or $y-w$ is not unit-regular for any unit $w \in R$. Let $\Omega$ be the set of all two-sided ideals $A$ of $R$ such that $1+x(y-w)$ is not a unit or $y-w$ is not unit-regular modulo $A$ for any unit $w+A \in R / A$. Obviously, $\Omega \neq \varnothing$. 
Analogously to the discussion in Lemma 1, we can find a two-sided ideal $Q$ of $R$ such that it is maximal in $\Omega$. Set $S=R / Q$. If $J(S) \neq 0$, then $J(S)=K / Q$ for some $K \supset Q$. Obviously, $S / J(S) \cong R / K$. By the maximality of $Q$, we have a unit $(v+Q)+J(S)$ such that $((1+x(y-v))+Q)+J(S)$ is a unit of $S / J(S)$ and $((y-v)+Q)+J(S)$ is unitregular in $S / J(S)$. Since units of $S / J(S)$ can be lifted modulo $J(S)$ because $S$ is an exchange ring, we may assume that $v+Q$ is unit of $S$. Similarly to Lemma 1 , we see that $(1+x(y-v))+Q=(m+Q)+(r+Q)$ for some $m+Q \in U(S), r+Q \in J(S)$. Thus, $(1+x(y-v))+Q$ is a unit of $S$. On the other hand, idempotents of $S / J(S)$ can be lifted modulo $J(S)$ because $S$ is an exchange ring. So we may assume that $((y-v)+Q)+$ $J(S)=((f+Q)+J(S))((u+Q)+J(S))$ with $f+Q=(f+Q)^{2} \in R / Q, u+Q \in U(S)$. Thus we can find some $t \in R$ with $t+Q \in J(S)$ such that $(y-(v-t))+Q=(f+$ $Q)(u+Q)$ is unit-regular in $S$. From $(1+x(y-(v-t)))+Q=((1+x(y-v))+Q)+$ $(x+Q)(t+Q)$ with $(1+x(y-v))+Q \in U(S)$ and $t+Q \in J(S)$, one easily checks that $(1+x(y-(v-t)))+Q$ is a unit of $S$. This contradicts the choice of $Q$. Therefore $J(S)=0$. The maximality of $Q$ implies that $S$ is indecomposable as a ring. By virtue of [14, Lemma 3.7], $S$ is a simple artinian ring. Assume that $R / Q \cong M_{n}(D)$, where $n$ is a positive integer and $D$ is a division ring. Since $R$ does not have $\mathbb{Z} / 2 \mathbb{Z}$ as a homomorphic image, we claim that $n \geq 2$, or $n=1$, and $D \nRightarrow \mathbb{Z} / 2 \mathbb{Z}$. Thus $R / Q$ satisfies unit 1 -stable range. From $((1+x y)+Q) S+((-x)+Q) S=S$, we have a unit $s+Q$ of $R / Q$ such that $(1+x(y-s))+Q=((1+x y)+Q)+((-x)+Q)(s+Q)$ is a unit of $S$. Since $S$ is simple artinian, $(y-s)+Q$ is unit-regular in $S$, a contradiction. Therefore the proof is complete.

THEOREM 7. Let $R$ be an exchange ring with primitive factors artinian. If $R$ does not have $\mathbb{Z} / 2 \mathbb{Z}$ as a homomorphic image, then $K_{1}(R) \cong U(R) / L_{1}(R)$.

Proof. Since $R$ is an exchange ring with primitive factors artinian, by virtue of Theorem 3, we know that $K_{1}(R) \cong U(R) / V(R)$. Let $b, c \in R$ with $p(b, c) \in U(R)$. In view of Lemma 6, there exists some $w \in U(R)$ such that $1+b(c-w) \in U(R)$ and $c-w=s$ unit-regular. Observe that

$$
\begin{aligned}
p(b, c) & =1+b c=1+b s+b w=1+b s+b(1+s b)(1+s b)^{-1} w \\
& =(1+b s)\left(1+b(1+s b)^{-1} w\right)=p(b, s) p\left(b(1+s b)^{-1}, w\right) .
\end{aligned}
$$

Likewise, we see that

$$
p(c, b)=p\left(w, b(1+s b)^{-1}\right)(1+s b)=p\left(w, b(1+s b)^{-1}\right) p(s, b) .
$$

By Lemma 5 and the fact that units commute $\bmod L_{1}(R)$, we see that

$$
\begin{aligned}
p(b, c) & =p(b, s) p\left(b(1+s b)^{-1}, w\right) \equiv p\left(b(1+s b)^{-1}, w\right) p(b, s) \\
& \equiv p\left(w, b(1+s b)^{-1}\right) p(s, b)=p(c, b) \quad\left(\bmod L_{1}(R)\right) .
\end{aligned}
$$

Hence, $L_{1}(R)=V(R)$ and we conclude that $K_{1}(R) \cong U(R) / L_{1}(R)$, as asserted.

COROLLARY 8. Let $R$ be an exchange ring with primitive factors artinian. If $2 \in U(R)$, then $K_{1}(R) \cong U(R)^{a b}$.

Proof. Let $e=e^{2} \in R$. Since $2 \in U(R)$, we have $e=1 / 2+(2 e-1) / 2$. Obviously, $((2 e-1) / 2)(4 e-2)=1$. By [9, Lemma 1.5], $1+e R(1-e) \subseteq U(R)^{\prime}$, and then $L_{1}(R)=U(R)^{\prime}$. 
On the other hand, $2 \in U(R)$ implies that $R$ does not have $\mathbb{Z} / 2 \mathbb{Z}$ as a homomorphic image. Using Theorem 7, we conclude that $K_{1}(R) \cong U(R) / L_{1}(R) \cong U(R) / U(R)^{\prime} \cong$ $U(R)^{a b}$, as desired.

COROLLARY 9. Let $R$ be an exchange ring of bounded index of nilpotence. If $2 \in U(R)$, then $K_{1}(R) \cong U(R)^{a b}$.

Proof. By [12, Proposition 2.1], we see that the primitive factors of $R$ are artinian. Thus the result follows from Corollary 8 .

ACKNOWLEDGEMENTS. It is a pleasure to thank the referee for thoroughly reading the article and for helpful suggestions.

This work is supported by the National Science Foundation of China, No. 19801012.

\section{REFERENCES}

[1] P. Ara, K. R. Goodearl, K. C. O'Meara, and E. Pardo, Separative cancellation for projective modules over exchange rings, Israel J. Math. 105 (1998), 105-137. MR 99g:16006. Zbl 908.16002.

[2] P. Ara, K. R. Goodearl, K. C. O'Meara, and R. Raphael, $K_{1}$ of separative exchange rings and $C^{*}$-algebras with real rank zero, Pacific J. Math. 195 (2000), no. 2, 261-275. CMP 1782 176. Zbl 991.71120.

[3] H. Chen, Units, idempotents and stable range conditions, to appear in Comm. Algebra.

[4] _ Rings with many idempotents, Int. J. Math. Math. Sci. 22 (1999), no. 3, 547-558. MR 2000k:16040. Zbl 992.03241.

[5] H. Chen and F. Li, Rings with many unit-regular elements, Chinese Ann. Math. Ser. A 21 (2000), no. 1, 27-32 (Chinese). CMP 1758 606. Zbl 946.19001.

[6] K. R. Goodearl, von Neumann Regular Rings, Monographs and Studies in Mathematics, vol. 4, Pitman, London, 1979. MR 80e:16011. Zbl 411.16007.

[7] K. R. Goodearl and P. Menal, Stable range one for rings with many units, J. Pure Appl. Algebra 54 (1988), no. 2-3, 261-287. MR 89h:16011. Zbl 653.16013.

[8] P. Menal, On $\pi$-regular rings whose primitive factor rings are artinian, J. Pure Appl. Algebra 20 (1981), no. 1, 71-78. MR 81k:16015. Zbl 457.16006.

[9] P. Menal and J. Moncasi, $K_{1}$ of von Neumann regular rings, J. Pure Appl. Algebra 33 (1984), no. 3, 295-312. MR 86i:18014. Zbl 541.16021.

[10] E. Pardo, Metric completions of ordered groups and $K_{0}$ of exchange rings, Trans. Amer. Math. Soc. 350 (1998), no. 3, 913-933. MR 98e:46088. Zbl 894.06007.

[11] J. R. Silvester, Introduction to Algebraic K-Theory, Chapman and Hall Mathematics Series, Chapman and Hall, London, 1981. MR 83f:18013. Zbl 468.18006.

[12] H.-P. Yu, On quasi-duo rings, Glasgow Math. J. 37 (1995), no. 1, 21-31. MR 96a:16001. Zbl 819.16001.

[13] _ Stable range one for exchange rings, J. Pure Appl. Algebra 98 (1995), no. 1, 105109. MR 96g:16006. Zbl 837.16009.

[14]_ On the structure of exchange rings, Comm. Algebra 25 (1997), no. 2, 661-670. MR 97k:16010. Zbl 873.16007.

Huanyin Chen: Department of Mathematics, Hunan Normal University, Changsha 410006, CHINA

E-mail address: chyzx1@sparc2 . hunnu.edu.cn

Fu-An Li: Institute of Mathematics, Chinese Academy of Sciences, Beijing 100080, CHINA

E-mail address: fa1@math08.math.ac.cn 


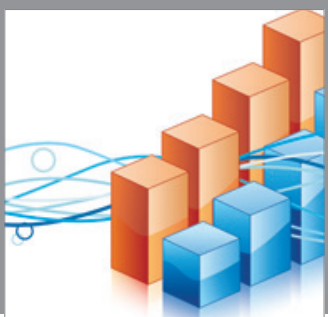

Advances in

Operations Research

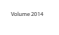

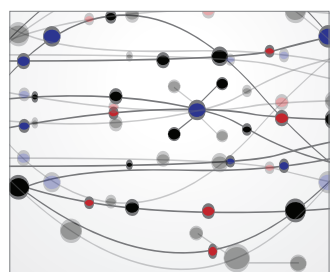

\section{The Scientific} World Journal
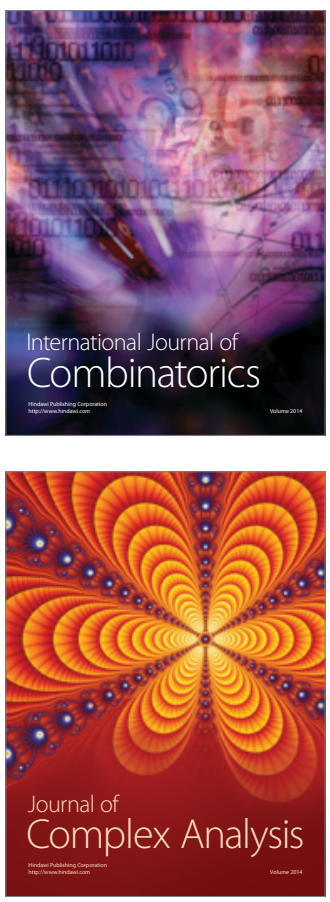

International Journal of

Mathematics and

Mathematical

Sciences
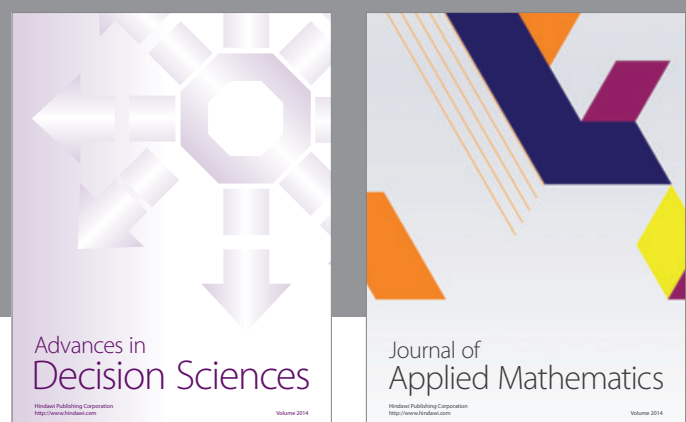

Journal of

Applied Mathematics
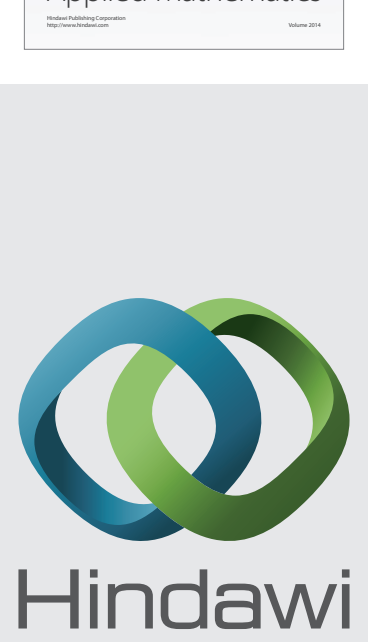

Submit your manuscripts at http://www.hindawi.com
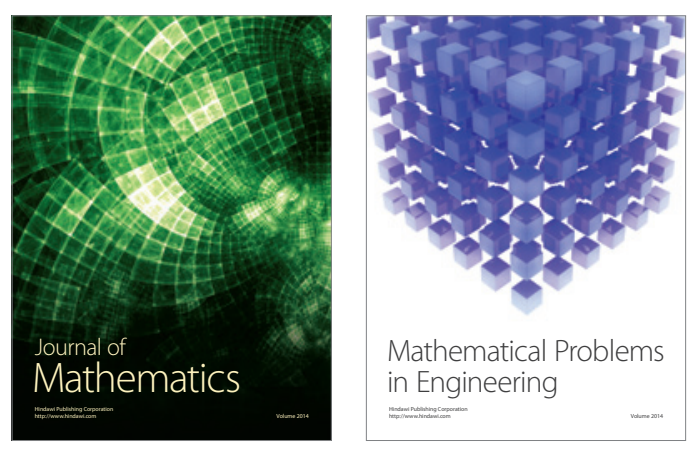

Mathematical Problems in Engineering
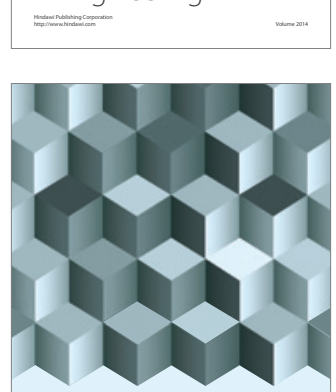

Journal of

Function Spaces
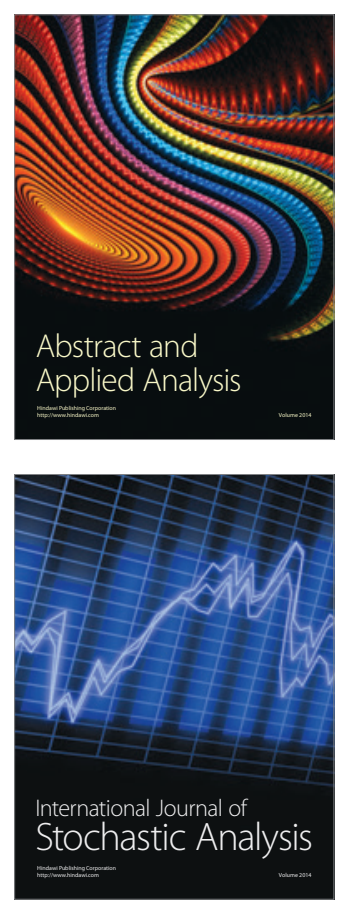

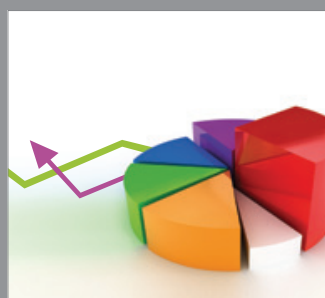

ournal of

Probability and Statistics

Promensencen
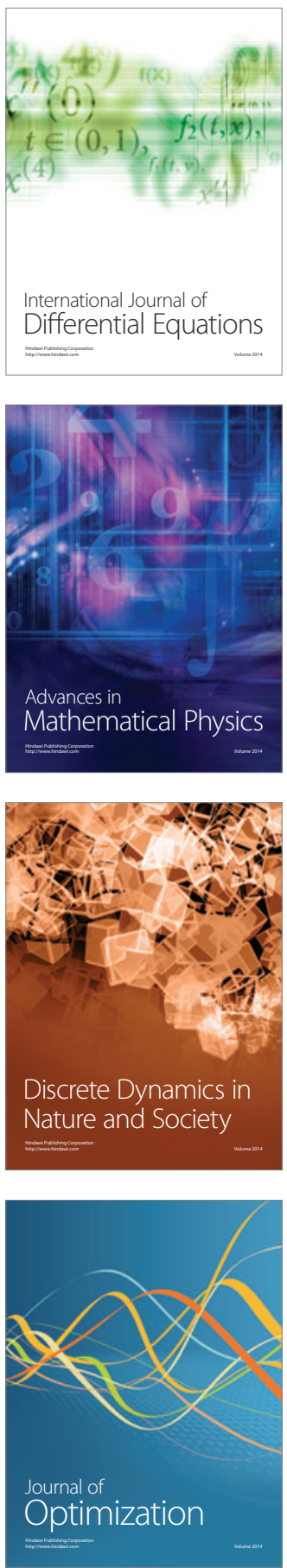